\title{
Probing the atmosphere of a solar-like star by galactic microlensing at high magnification
}

\author{
F. Abe ${ }^{1}$, D. P. Bennett ${ }^{2}$, I. A. Bond ${ }^{3}$, J. J. Calitz ${ }^{4}$, A. Claret ${ }^{5}$, K. H. Cook ${ }^{6}$, Y. Furuta ${ }^{1}$, A. Gal-Yam ${ }^{7}$, \\ J.-F. Glicenstein ${ }^{8}$, J. B. Hearnshaw ${ }^{9}$, P. H. Hauschildt ${ }^{10}$, D. Kent ${ }^{9}$, P. M. Kilmartin ${ }^{9}$, Y. Kurata ${ }^{1}$, K. Masuda ${ }^{1}$, \\ D. Maoz ${ }^{7}$, Y. Matsubara ${ }^{1}$, P. J. Meintjes ${ }^{4}$, M. Moniez ${ }^{1}$, Y. Muraki ${ }^{1}$, S. Noda ${ }^{1}$, E. O. Ofek ${ }^{7}$, K. Okajima ${ }^{1}$, \\ L. Philpott ${ }^{12}$, N. J. Rattenbury ${ }^{12}$, S. H. Rhie ${ }^{2}$, T. Sako ${ }^{1}$, D. J. Sullivan ${ }^{13}$, T. Sumi ${ }^{14}$, D. M. Terndrup ${ }^{15}$, \\ P. J. Tristram ${ }^{9}$, T. Yanagisawa ${ }^{1}$, and P. C. M. Yock ${ }^{12}$ \\ 1 Solar Terrestrial Environment Laboratory, Nagoya University, Nagoya 464-01, Japan \\ 2 Department of Physics, Notre Dame University, Notre Dame, IN 46556, USA \\ 3 Institute for Astronomy, University of Edinburgh, Edinburgh, EH9 3HJ, UK \\ ${ }^{4}$ Department of Physics, University of the Free State, Bloemfontein, South Africa \\ 5 Instituto de Astrofísica de Andalucía, CSIC, Apartado 3004, 18080 Granada, Spain \\ ${ }^{6}$ Institute for Geophysics and Planetary Physics, Lawrence Livermore National Laboratory, CA 94563, USA \\ 7 School of Physics and Astronomy, Tel-Aviv University, Tel-Aviv 69978, Israel \\ ${ }^{8}$ CEA, DSM, DAPNIA, Centre d'Études de Saclay, 91191 Gif-sur-Yvette Cedex, France \\ 9 Department of Physics and Astronomy, University of Canterbury, Private Bag 4800, Christchurch, New Zealand \\ ${ }^{10}$ Universität Hamburg, Sternwarte, Gojenbergsweg 112, 21029 Hamburg, Germany \\ ${ }^{11}$ Laboratoire de l'Accélérateur Linéaire, IN2P3 CNRS, Université de Paris-Sud, 91405 Orsay Cedex, France \\ 12 Faculty of Science, University of Auckland, Private Bag 92019, Auckland, New Zealand \\ 13 School of Chemical and Physical Sciences, Victoria University, PO Box 600, Wellington, New Zealand \\ 14 Department of Astrophysical Sciences, Princeton University, Princeton NJ 08544, USA \\ 15 Department of Astronomy, Ohio State University, Columbus OH 43210, USA
}

Received 2 July 2003 / Accepted 13 Octobre 2003

\begin{abstract}
We report a measurement of limb darkening of a solar-like star in the very high magnification microlensing event MOA 2002-BLG-33. A 15 hour deviation from the light curve profile expected for a single lens was monitored intensively in $V$ and $I$ passbands by five telescopes spanning the globe. Our modelling of the light curve showed the lens to be a close binary system whose centre-of-mass passed almost directly in front of the source star. The source star was identified as an F8-G2 main sequence turn-off star. The measured stellar profiles agree with current stellar atmosphere theory to within $\sim 4 \%$ in two passbands. The effective angular resolution of the measurements is $<1 \mu$ as. These are the first limb darkening measurements obtained by microlensing for a Solar-like star.
\end{abstract}

Key words. techniques: high angular resolution - techniques: gravitational microlensing - stars: limb darkening stars: individual: MOA 2002-BLG-33

\section{Introduction}

In gravitational microlensing events binary lenses produce bounded regions of high magnification, known as caustics, on the magnification map projected onto the source plane (Schneider \& Weiss 1986). The steep magnification gradients associated with these caustic curves may be utilised to resolve the surfaces of background source stars as they move across them. Observations of this effect have been used to measure limb darkening in Galactic Bulge K giant stars (Albrow et al. 1999; Albrow et al. 2000; Fields et al. 2003), a G/K sub-giant

Send offprint requests to: I. A. Bond, e-mail: iab@roe.ac.uk
(Albrow et al. 2001a), and an A dwarf in the Small Magellanic Cloud (reporting an angular resolution of a few nas, Afonso et al. 2000).

In this Letter we present observations of the very high magnification microlens event MOA 2002-BLG-33. In this event, the centre-of-mass of the close binary lensing system moved into near perfect alignment with a Solar-like source star. The resulting high magnification provided ideal conditions for monitoring the source as it transited the central caustic. Our observations yielded the most precise limb darkening measurements obtained by microlensing for a non-giant star and the first such measurements for a Solar-like star other than the Sun itself. 


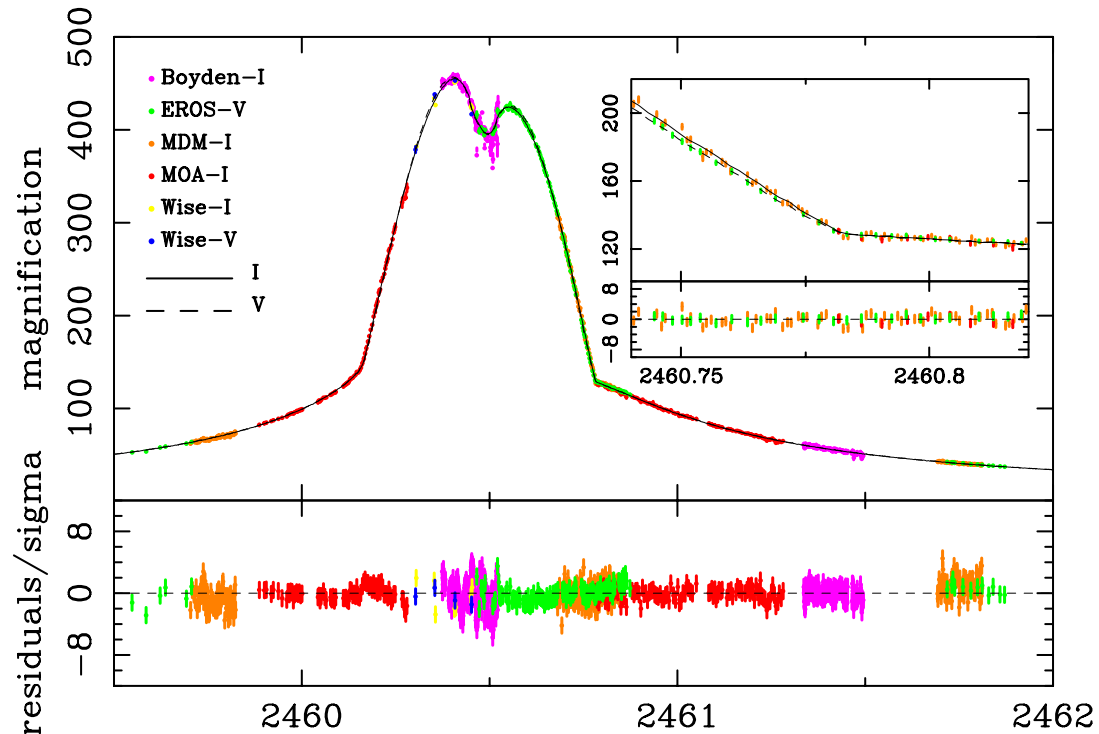

HJD - 2450000
Fig. 1. Observed light curve, best fitting model, and residuals from the model for the peak of MOA 2002-BLG-33. The total dataset consists of 473 measurements in the $I$ band from Boyden $1.5 \mathrm{~m}$ telescope (South Africa), 186 points in $V_{\mathrm{E}}$ from EROS $1 \mathrm{~m}$ (La Silla), 260 points in $I$ from MDM $2.4 \mathrm{~m}$ (Kitt Peak), 480 points in $I$ from MOA $0.6 \mathrm{~m}$ (New Zealand), and 21 points in $I$ and 10 points in $V$ from Wise $1 \mathrm{~m}$ (Israel). The inset shows the separation of the light curves in the $V_{\mathrm{E}}$ and $I$ bands caused by limb reddening.

\section{Light curve of MOA 2002-BLG-33}

The event MOA 2002-BLG-33 was first alerted by the MOA collaboration on 2002 June 18 in its ongoing microlensing alert program (Bond et al. 2001). About 48 hours before its time of peak amplification on 2002 July 4, MOA identified this event as a high magnification event in progress and a second alert was issued. Subsequently, an intensive campaign of follow-up observations was carried out using a number of telescopes circling the globe (Fig. 1). Photometry of the event was derived using difference imaging analysis applied to the CCD images. This procedure was carried out separately on each passband of each telescope resulting in six sets of time series photometry. These are shown in Fig. 1 where they have been normalised to the microlensing light curve using the best fitting model (described below).

The M-shaped feature on the light curve results from the source star entering and exiting the central caustic curve produced by a binary lens. The detailed shape of the light curve depends on the characteristics of the lens, the trajectory of the source star, and the radius and limb darkening profile of the source star. The parameters associated with the binary lens are the lens mass ratio $M_{1} / M_{2}$, the separation $a$ of the two lenses, the Einstein radius crossing time $t_{\mathrm{E}}$, the source trajectory impact parameter, $u_{0}$, with respect to the centre-of-mass of the lens, the time $t_{0}$ when the source is closest to the centre of mass of the lens, and the angle, $\phi$, between the source trajectory and the lens components.

The source star is parametrized by its radius $r_{\mathrm{s}}$ and an appropriate limb darkening model. For this we used the squareroot law (Claret 2000)

$\frac{I_{\lambda}(\theta)}{I_{\lambda}(0)}=1-c_{\lambda}(1-\cos \theta)-d_{\lambda}(1-\sqrt{\cos \theta})$

where $c_{\lambda}$ and $d_{\lambda}$ are the wavelength dependent limb darkening coefficients.

Model binary microlensing light curves were generated numerically using an inverse ray tracing technique, implemented on a cluster computer (Rattenbury et al. 2002). The inverse ray tracing technique naturally accommodates the finite size of the source star and the limb darkening profiles across its face (Wambsganss 1997). To find the best fitting model light curve, we implemented a $\chi^{2}$ minimisation technique using a Metropolis Hastings Markov chain Monte Carlo procedure (Ford 2003). This avoids the problem of traditional minimisation techniques which can be fooled by false minima due to numerical noise in the simulated light curves.

The best fitting theoretical curve is shown in Fig. 1. A total of 11 physical parameters plus 6 flux normalisation parameters (one for each passband-telescope set) are required to describe the light curve ${ }^{1}$. The best fitting physical parameters are given in Table 1. The fitting procedure yielded correlated values for the limb darkening coefficients. In Table 1, we report the coefficients $\left(c_{\lambda}^{\prime}, d_{\lambda}^{\prime}\right)$, which are related to the coefficients in Eq. (1) through the phase space rotation $c_{\lambda}^{\prime}=c_{\lambda} \cos 33^{\circ}+d_{\lambda} \sin 33^{\circ}$ and $d_{\lambda}^{\prime}=d_{\lambda} \cos 33^{\circ}-c_{\lambda} \sin 33^{\circ}$. In this representation, the $d_{\lambda}^{\prime}$ term contributes a small correction to $I_{\lambda}(\theta) / I_{\lambda}(0)$. The limb darkening parameters allow a colour map of the face of the source star to be drawn. This is depicted in Fig. 2 along with its passage through the caustic.

Our best best fitting model light curve and the associated caustic correspond to a close binary lensing system. Dominik (1999) found that qualitatively similar caustic curves can be produced by lensing configurations that differ greatly. Caustics similar in shape to the one shown in Fig. 2 can be produced by lenses comprised of wide binary systems. Our best fitting wide binary solution for our observations of MOA 2003-BLG-33 yielded a mass ratio corresponding to that of a giant planet orbiting a stellar mass object. While this configuration qualitatively produced a short duration M-shaped amplification profile, it could not reproduce the observed light curve profile at a level demanded by the precise, densely sampled photometry

\footnotetext{
1 Blending parameters were not required because difference imaging analysis removes the effects of blending due to nearby neighbours and the lens itself.
} 
Table 1. Microlensing parameters of MOA 2002-BLG-33. Here $r_{\mathrm{E}}$ denotes the Einstein radius of the lens, as determined by its total mass, $M_{1}+M_{2}, D_{\mathrm{OL}}$ and $D_{\mathrm{OS}}$ the distances to the lens and source stars. The errors are $2 \sigma$ limits calculated as in Avni (1976).

\begin{tabular}{lll}
\hline \hline Parameter & \multicolumn{2}{c}{ Value } \\
\hline Lens mass ratio & $M_{1} / M_{2}$ & $0.54 \pm 0.20$ \\
Lens separation & $a$ & $0.11 \pm 0.01 r_{\mathrm{E}}$ \\
Einstein time & $t_{\mathrm{E}}$ & $50.7 \pm 1.0$ day \\
Position angle & $\phi$ & $112^{\circ} \pm 2^{\circ}$ \\
Source radius & $r_{\mathrm{S}}$ & $(2.25 \pm 0.05) \times 10^{-3} r_{\mathrm{E}} D_{\mathrm{OS}} / D_{\mathrm{OL}}$ \\
Impact parameter & $u_{0}$ & $(4.29 \pm 0.09) \times 10^{-4} r_{\mathrm{E}}$ \\
Centre time & $t_{0}$ & $\mathrm{HJD} 2452460.496 \pm 0.004$ \\
Limb darkening & $c_{I}^{\prime}$ & $0.41 \pm 0.04$ \\
& $d_{I}^{\prime}$ & $-0.08 \pm 0.65$ \\
& $c_{V_{\mathrm{E}}}^{\prime}$ & $0.49 \pm 0.02$ \\
& $d_{V_{\mathrm{E}}}^{\prime}$ & $1.15 \pm 0.56$ \\
\hline
\end{tabular}

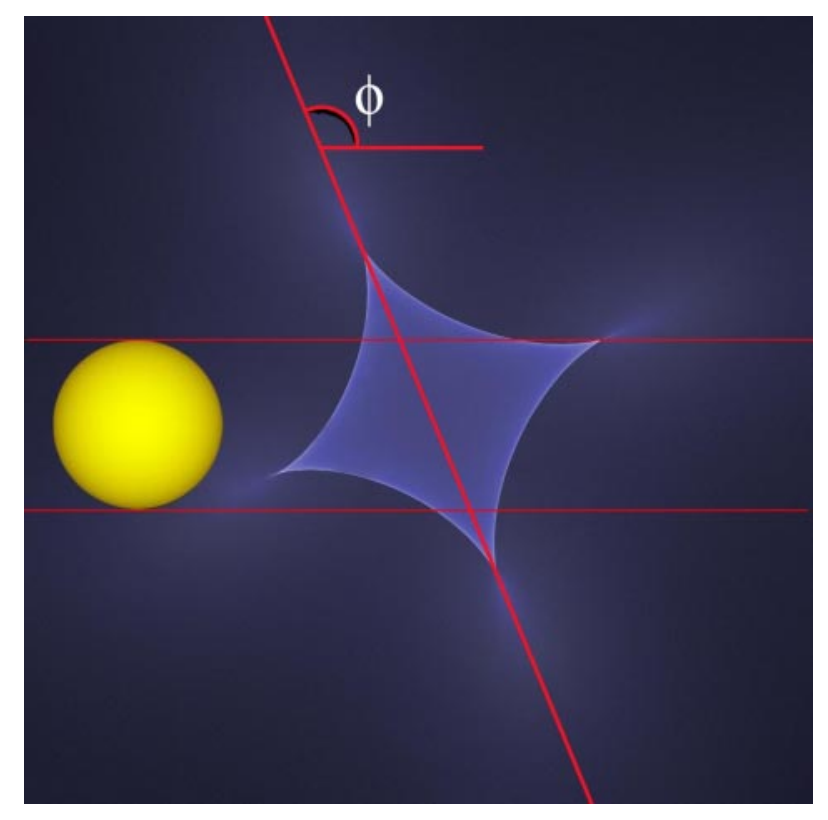

Fig. 2. The passage of the source star of MOA 2002-BLG-33 through the lens-caustic. The magnification map of the lens is represented in blue, with regions of higher magnification represented by lighter shading. The caustic is the diamond shaped line of very high magnification. The red lines indicate the track of the source star relative to the caustic. The position angle of the lens binary system with respect to the source trajectory is denoted by $\phi$. The angular diameter of the source star would be $2-4 \mu$ as.

we obtained. Only the above close binary model gave an acceptable fit to the data.

Unfortunately, neither the rotation period of the binary lens, nor the plane of rotation, was able to be determined from the data. We estimated the contribution of binary rotation to our measurements of limb darkening as follows. For typical values of the lens parameters, the rotation period lies in the range 100 200 days. During the caustic crossing shown in Fig. 2, the lens is thus expected to rotate through $1^{\circ}-2^{\circ}$. Light curves were computed for rotating lenses with various periods of rotation, but with the plane of rotation held fixed and perpendicular to the lens-source axis, to maximise the effect. It was found that periods of rotation $<150$ days yielded light curves that were inconsistent with the data at a level greater than $2 \sigma$. Limb darkening coefficients, and stellar profiles, were then computed, in the static approximation, for a simulated dataset with a rotation period of 150 days. It was found that the computed stellar profiles differed from the inputted profiles by approximately $2 \times$ the $2 \sigma$ statistical uncertainty of the profiles computed without rotation. The effect of rotation was therefore allowed for, in the present work, by doubling the errors in the stellar profiles calculated in the static approximation.

\section{The surface of the source star}

We used colour measurements to identify the source star type in MOA 2003-BLG-33. The baseline (i.e. unmagnified) flux of the source star was determined by from the fluxes measured by the MDM and Wise telescopes at high magnification. The instrumental magnitudes were calibrated using the catalogue of Udalski et al. (2002). The magnitudes thus determined in the $V$ and $I$ bands (Landolt system) were $m_{V}=19.94 \pm 0.05$ and $m_{I}=17.96 \pm 0.07$. Assuming a distance to the source star of $7 \pm 2 \mathrm{kpc}$, and absorption and reddening due to galactic dust with $A_{I}=1.18 \pm 0.05 \mathrm{mag}$ and $E(V-I)=1.24 \pm 0.05$ (Sumi et al. 2003; Udalski 2003), we derive its absolute magnitude $M_{I}=2.6 \pm 0.6$ and colour index $V-I=0.74 \pm 0.10$. These values identify the source star as an F8-G2 main-sequence turnoff star with age approximately 3-10 Gy and temperature in the range 6200-5800 K (Girardi et al. 2002), i.e. a solar-like star that has just commenced evolving away from the main sequence, or is just about to do so.

Limb-darkening coefficients have been calculated for the ATLAS and PHOENIX models of stellar atmospheres for a wide variety of stars (Claret 2000). The limb darkening coefficients, $\left(c_{\lambda}^{\prime}, d_{\lambda}^{\prime}\right)$, for the ATLAS model of a star with $T_{\text {eff }}=6000 \mathrm{~K}$, surface gravity $\log g=4.0$, and metallicity $[\mathrm{M} / \mathrm{H}]=-0.3$ are $(0.363,0.459)$ for the $I$-band and $(0.461$, $0.394)$ for the $V_{\mathrm{E}}$-band ${ }^{2}$. These compare favourably with the measured values in in Table 1 that were derived from our observations without including the effect of rotation described above. Figure 3 shows the theoretical intensity profiles for the range of possible star types identified for MOA 2003-BLG-33, and the intensity profiles derived from the measurements with the effect of rotation included. It is apparent that the ATLAS and PHOENIX profiles are confirmed at the $95 \%$ confidence level to within about $4 \%$. The precision of the present measurements resulted from the fact that the magnification was high throughout the caustic crossing, and that it could therefore be monitored accurately everywhere, in particular at the end points which are most sensitive to limb darkening and reddening, as

\footnotetext{
${ }^{2}$ For the $V_{\mathrm{E}}$-band, the average values given by Claret (2000) in the $V$ and $R$ bands were used, to allow for the extended red transmission of the EROS $V$ filter, and also the pre-filtering effect of galactic dust.
} 

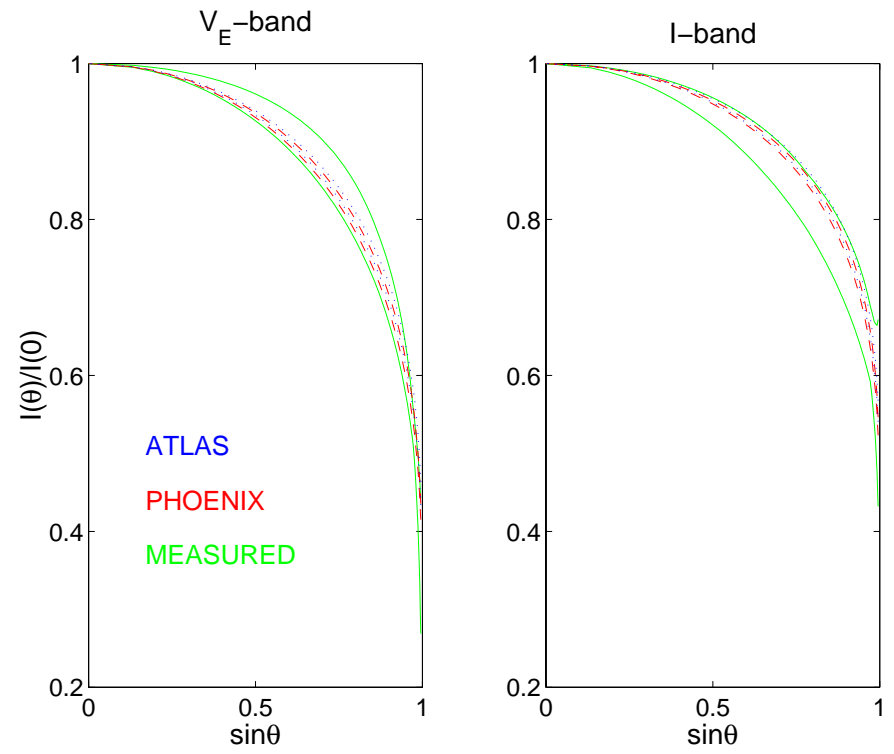

Fig. 3. Comparison of the measured profiles at visual (left) and infrared (right) wavelengths of the source star of MOA 2002-BLG-33, with predictions of the ATLAS and PHOENIX models of stellar atmospheres (Claret 2000). 95\% confidence limits are shown for the measured profiles. The $x$-axis variable, $\sin \theta$, is the distance from the centre of the face of the star normalised to the star's radius. For the model atmospheres, the temperatures have been set at $5800 \mathrm{~K}$ (lower curves) or $6200 \mathrm{~K}$ (upper curves), the metallicity at $[\mathrm{M} / \mathrm{H}]=-0.3$ (Tiede et al. 1995), and the surface gravity $\log g=4.0$.

shown in Fig. 1. The effective angular resolution of our observations of MOA $2002-\mathrm{BLG}-33$ is $<1 R_{\odot} / 7 \mathrm{kpc}$, i.e. $<1 \mu$ as.

Our results appear to be in agreement with those expected by stellar atmosphere models for the source star type identified here. Limb darkening measurements for another solar-like star, and consistent with those reported here, have been obtained by Deeg et al. (2001) using a planetary transit technique. The only other observations by gravitational microlensing of limb darkening on a main sequence star were obtained for the A dwarf source star in MACHO 1997-SMC-1 (Afonso et al. 2000). These were only partly in agreement with stellar atmosphere theory (Claret 2000). Limb darkening measurements in agreement with theoretical expectations have been reported for a G/K subgiant source in OGLE 1999-BLG-23 (Albrow et al. 2001a), and for the K giant source stars in MACHO 1997BLG-28 (Albrow et al. 1999) and in MACHO 1997-BLG-41 (Albrow et al. 2000). On the other hand, recent results on source star in EROS 2000-BLG-5, also a K giant, appear in strong disagreement with stellar atmosphere theory (Afonso et al. 2001; Fields et al. 2003) whereas no such discrepancy was observed in $\mathrm{H}_{\alpha}$ equivalent width variations obtained in spectroscopic observations of the same event (Albrow et al. 2001b).

\section{Conclusion and outlook}

We have shown how a high magnification microlensing event, with the lens being a close binary system, can be utilised to profile the atmospheres of a dwarf star. Such stars, even if nearby, cannot be resolved using conventional techniques. Thus the high magnification microlensing technique may prove useful until new, major programmes with the Very Large Telescope Interferometer and the Keck Interferometer become fully operational (Segransan et al. 2003; Domiciano de Souza et al. 2003). We note that although some previous measurements using microlensing techniques yielded possible evidence for significant departures from conventional stellar atmosphere theory, no such evidence was obtained in the present measurements. Indeed, as the star under study here was quite similar to the Sun, no such departure was expected.

Acknowledgements. We thank the referee, J.-P. Beaulieu, for helpful suggestions in improving the presentation of this manuscript. We thank P. Dobcsanyi, B. Donovan, G. Gimel'farb, A. Gould, A. Gyekye, P. Leonne and S. Peale for assistance, the Space Telescope Science Institute for Director's Discretionary time on the HST, the Marsden Fund of New Zealand, the Department of Education, Science and Culture of Japan, the NSF (USA), and the University of Auckland for financial support, and also the Pôle Scientifique de Modélisation Numérique (ENS-Lyon), the Norddeutscher Verbund für Höch und Höchstleistungsrechnen (HLRN), and the NERSC for computer time. This work was performed under the auspices of the U.S. Department of Energy, National Nuclear Security Administration by the University of California, Lawrence Livermore National Laboratory under contract No. W-7405-Eng-48.

\section{References}

Afonso, F., Alard, C., Albert, J. N., et al. 2000, ApJ, 532, 340

Afonso, F., Alard, C., Albert, J. N., et al. 2001, A\&A, 378, 1014

Albrow, M. D., Beaulieu, J.-P., Caldwell, J. A. R., et al. 1999, ApJ, 522,1011

Albrow, M., An, J., Beaulieu, J.-P., et al. 2000, ApJ, 534, 894

Albrow, M., An, J., Beaulieu, J.-P., et al. 2001a, ApJ, 549, 759

Albrow, M., An, J., Beaulieu, J.-P., et al. 2001b, ApJ, 550, L173

Avni, Y. 1976, ApJ, 210, 642

Bond, I. A., Abe, F., Dodd, R. J., et al. 2001, MNRAS, 327, 868

Claret, A. 2000, A\&A, 363, 1081

Deeg, H. J., Garrido, R., \& Claret, A. 2001, New Astron., 6, 51

Domiciano de Souza, A., Kevella, P., Jackov, S., et al. 2003, A\&A, 407, L47

Dominik, M. 1999, A\&A, 349, 108

Fields, D. L., Albrow, M. D., An, J., et al. 2003, ApJ, 596, 1305

Ford, E. B. 2003, AJ, submitted [astro-ph/0305441]

Girardi, L., Bertelli, G., Bressan, A., et al. 2002, A\&A, 391, 195

Rattenbury, N. J., Bond, I. A., Skuljan, J., \& Yock, P. C. M. 2002, MNRAS, 335, 159

Schneider, P., \& Weiss, A. 1986, A\&A, 164, 237

Segransan, D., Kervella, P., Forveille, T., \& Queloz, D. 2003, A\&A, 397, L5

Sumi, T., Abe, F., Bond, I. A., et al. 2003, ApJ, 591, 204

Tiede, G. P., Frogel, J. A., \& Terndrup, D. M. 1995, AJ, 110, 2788

Udalski, A., Szymanski, M., Kubiak, M., et al. 2002, Acta Astron., 52, 217

Udalski, A. 2003, ApJ, 590, 284

Wambsganss, J. 1997, MNRAS, 284, 172 